\title{
Clinical Features of Bloodstream Infections Associated with Peripheral Versus Central Venous Catheters
}

\author{
Keita Tatsuno (D) - Mahoko Ikeda - Yoshitaka Wakabayashi • \\ Shintaro Yanagimoto · Shu Okugawa · Kyoji Moriya
}

Received: June 11, 2019 / Published online: July 31, 2019

(C) The Author(s) 2019

\begin{abstract}
Introduction: This study aimed to compare the clinical characteristics and prognoses of central venous catheter-associated bloodstream infections (CVC-BSIs) with peripheral venous catheter-associated BSIs (PVC-BSIs).

Methods: This retrospective observational study was conducted between April 2011 and March 2013 at a teaching hospital in Tokyo, Japan. Adult patients who developed CVC-BSIs and PVC-BSIs more than 2 days after admission were included. Patients with both CVC-BSIs and PVC-BSIs were excluded. Clinical characteristics of patients with CVC-BSIs and PVC-BSIs were obtained from medical records, and 30-day allcause mortality was measured as the clinical outcome.
\end{abstract}

Enhanced Digital Features To view enhanced digital features for this article go to https://doi.org/10.6084/ m9.figshare.8961689.

K. Tatsuno $(\varangle) \cdot$ M. Ikeda $\cdot$ K. Moriya

Department of Infection Control and Prevention,

The University of Tokyo Hospital, 7-3-1, Hongo,

Bunkyo-ku, Tokyo, Japan

e-mail: tatsuno-tky@umin.ac.jp

Y. Wakabayashi · S. Yanagimoto · S. Okugawa ·

K. Moriya

Department of Infectious Diseases, The University of Tokyo Hospital, 7-3-1, Hongo, Bunkyo-ku, Tokyo, Japan
Results: We enrolled 124 PVC-BSI cases and 110 CVC-BSI cases. Median age, age-adjusted Charlson score, Sequential Organ Failure Assessment score, sex, and ward type at BSI onset did not differ significantly between the two groups. The median duration of catheter indwelling was significantly shorter in the PVCBSI group than in the CVC-BSI group. Staphylococcus aureus and Gram-negative bacilli infections were more frequent and coagulasenegative staphylococci (CNS) and Candida spp. infections were less frequent in the PVC-BSI group than in the CVC-BSI group. The prevalence of oxacillin resistance among causative $S$. aureus and CNS, 30-day all-cause mortality, and appropriateness of empirical and definitive antimicrobial therapies did not differ significantly between the two groups.

Conclusion: The pathogen species distribution varies between PVC-BSIs and CVC-BSIs. However, all-cause mortality does not differ between the two groups. PVCs are not safer than CVCs with respect to BSIs; therefore, it is necessary to use similar precautions relevant to CVC use in order to avoid unnecessary use of PVCs.

Keywords: Bloodstream infection; Central venous catheter; Mortality; Peripheral catheter 


\section{INTRODUCTION}

Venous catheters are among the most widely used medical devices and are indispensable for medical care. However, they can also lead to serious complications by causing bloodstream infections (BSIs). Therefore, it is crucial for patient safety to prevent catheter-related BSIs (CRBSIs) in hospital settings because they are not only associated with an increased hospital stay and extra attendant costs but also with increased mortality [1-3].

Most CRBSIs are related to the presence of central venous catheters (CVCs) rather than peripheral venous catheters (PVCs) [4]. The incidence rate of PVC-BSIs is estimated to be $0.05-0.5$ per 1000 device days, which is far less than the incidence rate of CVC-BSIs [5-7]. However, PVCs are more frequently used than CVCs in clinical settings [8, 9], and, therefore, the absolute number of PVC-BSI cases is not negligible.

CVC-BSIs have been well studied for their prevalence, pathogen species distribution, and major complications because their incidence rate is higher than that of PVC-BSIs. Only a few studies have focused specifically on the pathogen species distribution and prognosis in PVCBSIs, despite the more frequent PVC use compared to $\mathrm{CVC}$ use and potential for serious infections $[6,9,10]$. Considering that PVC-BSIs may be quite different from CVC-BSIs, some studies have focused on PVC-associated phlebitis or exit-site infections [11-15]. However, PVC-BSIs and phlebitis are not synonymous. In this study, we have compared the clinical characteristics and prognoses of CVC-BSIs and PVC-BSIs to further our understanding of their relative clinical impacts.

\section{METHODS}

\section{Study Setting}

This study was performed at the University of Tokyo Hospital [a 1210-bed tertiary care teaching hospital including 34 intensive care unit (ICU) beds for adults] in Tokyo, Japan, from April 2011 through March 2013. The infection control teams consisted of full-time infection control and infectious disease physicians and nurses.

\section{Study Design}

This was a retrospective observational cohort study. The enrolled cases were selected from those that involved patients with positive blood culture results. Patients with PVC-BSIs and CVC-BSIs were enrolled based on the criteria given below.

\section{Inclusion Criteria}

The bloodstream infection occurred more than 2 days after admission. CRBSI was determined when (1) a recognized pathogen was cultured from one or more blood cultures, and the organism was not related to infection at another site, or (2) for common commensal bacteria, such as coagulase-negative staphylococci (CNS), at least two consecutive positive blood cultures were obtained, with clear resolution of clinical symptoms after catheter withdrawal. CVC-BSI was defined according to the surveillance definition given by the Centers for Disease Control and Prevention National Healthcare Safety Network [16]. A patient with PVC who met the above criteria for bloodstream infection with a recognized pathogen or with commensal bacteria was defined as having a PVC-BSI. If more than two episodes occurred after the treatment of BSI was completed, they were enrolled as separate cases of CRBSI.

\section{Exclusion Criteria}

Patients who (1) were $<18$ years of age at the time of detection of positive blood cultures, (2) had both CVC and PVC, (3) had CVC-BSI associated with intravenous hyperalimentation therapy at home, and (4) had other venous catheters (such as peripherally inserted central catheter, hemodialysis catheter, central venous access port, Hickman-type catheters, or arterial catheters) along with a PVC or CVC were excluded from the study. 


\section{Data Collection and Definitions}

Data of the following clinical characteristics were obtained from medical records: age, sex, comorbidities, length of catheter indwelling, length of hospitalization, ward type on the onset day (medicine, surgery, emergency department, or ICU), receipt of mechanical ventilation, and severity of illness at BSI onset which was calculated using the Sequential Organ Failure Assessment (SOFA) score [17]). To calculate the age-adjusted Charlson score for mortality risk due to comorbid conditions $[18,19]$, the following comorbidities were documented: myocardial infarction, congestive heart failure, peripheral vascular disease, cerebrovascular disease, dementia, chronic obstructive pulmonary diseases, connective tissue disease, peptic ulcer disease, diabetes mellitus, chronic kidney disease, hemiplegia, leukemia, malignant lymphoma, solid tumor, liver disease, and acquired immune deficiency syndrome. The day of catheter removal, day of antibiotic regimen initiation, and the day of initiation and duration of appropriate antimicrobial therapy were all confirmed from the medical records. The first day was defined as the initial day that blood cultures were taken from the patient. Appropriate antimicrobial therapy was defined as systemic administration of at least one antimicrobial agent to which the causative pathogen showed susceptibility in vitro. The 30-day all-cause mortality was measured as the clinical outcome. Estimates of incidence rates of CRBSIs were calculated as episodes of BSI per 1000 patient-days.

\section{Microbiological Analysis}

Blood culture specimens were inoculated into BACTEC standard culture bottles in the BACTEC 9000 system (Becton Dickinson, Franklin Lakes, NJ, USA). The identification and susceptibility tests were performed using a WalkAway system (Siemens, Berlin, Germany). The broth microdilution method was used for susceptibility testing, and the results were interpreted according to the Clinical Laboratory Standards Institute guidelines (M100-S19).

\section{Statistical Analysis}

For continuous variables, mean values were compared using the two-sample Student's $t$ test for independent samples. The Mann-Whitney $U$ test was used when variables were skewed. Differences in proportion were compared using the Chi square test or Fisher's exact test, as appropriate. The Kaplan-Meier survival analysis and log-rank test were used to estimate the probability of survival after the onset of BSI. All $p$ values for these tests were two-tailed, and values of $p<0.05$ were considered statistically significant. All statistical analyses were performed using GraphPad Prism (v.5.0; GraphPad Software, San Diego, CA, USA). The study was approved by the ethics committee of Graduate School of Medicine and Faculty of Medicine, The University of Tokyo (\#3538). The requirement for written informed consent was waived due to the observational retrospective nature of the study.

\section{RESULTS}

\section{Case Characteristics}

During the study period, a total of 1,236 bacteremia cases were identified, of which 414 were associated with CRBSIs. Of these, 166 were PVCBSIs, 183 were CVC-BSIs, and 65 were caused due to other intravascular devices. Finally, 124 cases met the criteria for PVC-BSIs and 110 for CVC-BSIs (Fig. 1). The 47 cases of BSIs (30 CVC-BSIs and 27 PVC-BSIs) involved 21 patients, each with two or three episodes of BSI. The estimations of incidence rates of PVC-BSIs and CVC-BSIs were 0.17 and 0.15 per 1000 patient-days, respectively. The median age, age-adjusted Charlson scores, SOFA scores, sex distribution, and ward types were similar for both the groups. The median length of hospitalization and catheter indwelling times were significantly shorter in the PVC-BSI group than in the CVC-BSI group (Table 1).

\section{Pathogens Responsible for CVC-BSIs and PVC-BSIs}

The distribution of the causative pathogens differed between the CVC-BSI and PVC-BSI 


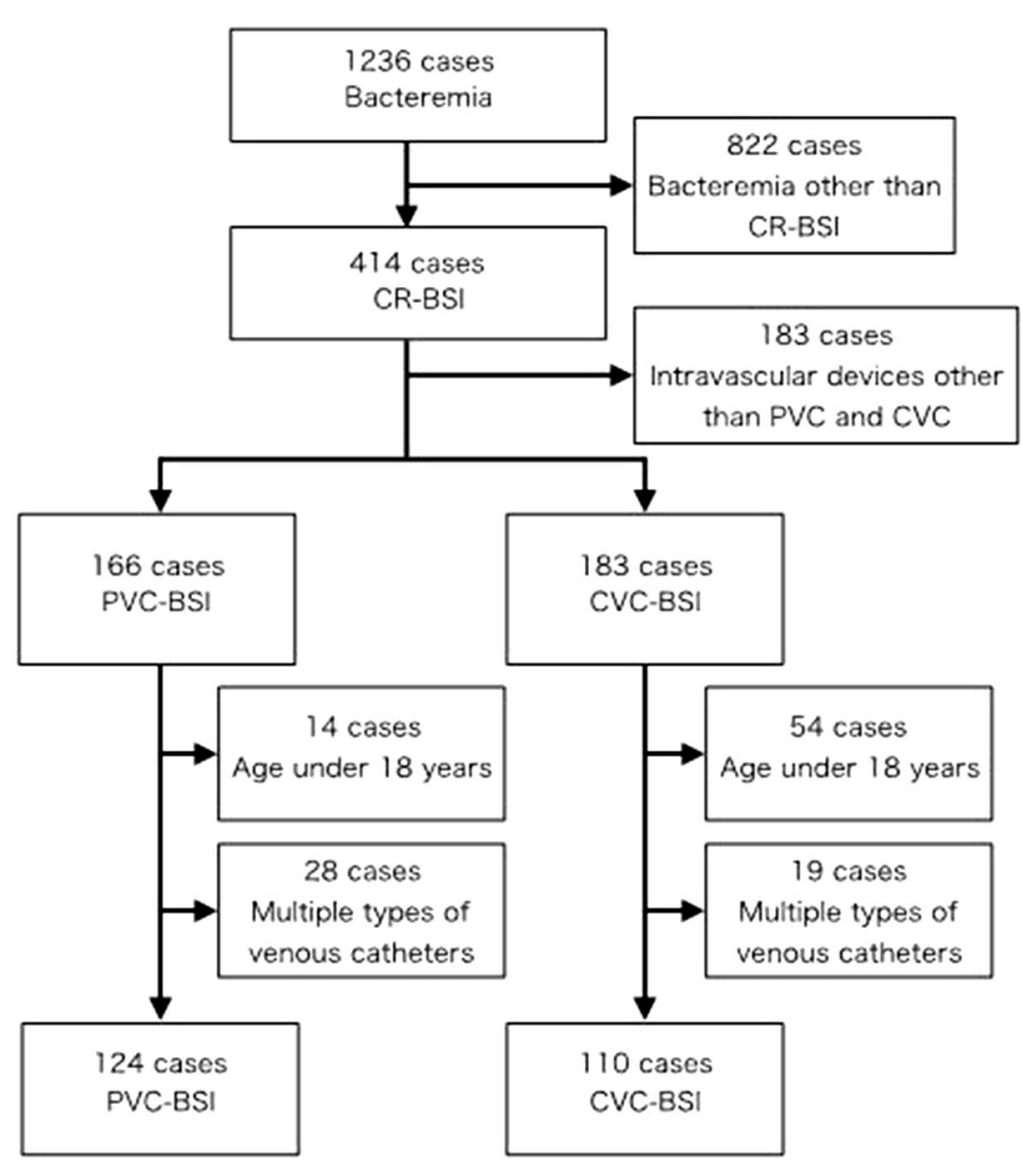

Fig. 1 Patient selection. The flow chart shows the selection of patients with peripheral venous catheter-associated and central venous catheter-associated bloodstream infections

groups. Staphylococcus aureus and Gram-negative bacilli infections were more frequent, and coagulase-negative staphylococci (CNS) and Candida spp. infections were less frequent in the PVC-BSI group than in the CVC-BSI group. The prevalence of oxacillin resistance of both $S$. aureus and CNS did not differ between the two groups (Table 2).

\section{Therapeutic Approach}

Catheter removal after BSI onset occurred earlier in the PVC-BSI group than in the CVC-BSI group (Table 3). While empirical treatment regimens and their appropriateness were comparable between the two groups, definitive treatment regimens differed significantly between the two groups because of the differences in the distribution of causative pathogens. In total, 33 patients in the PVC-BSI group and 15 in the CVC-BSI group received no antibiotics at the time of blood culture. No patient without antibiotic treatment died within 30 days of blood culture, even though 4 and 3 patients from the PVC-BSI and CVC-BSI groups, respectively, showed positive blood culture results.

\section{Clinical Outcomes}

The all-cause mortality at 30 days was $12.0 \%$ (14 patients) for the PVC-BSI group and 12.8\% (14 patients) for the CVC-BSI group ( $p=0.883$, logrank test; Fig. 2). Survival rates did not differ significantly between the two groups. 
Table 1 Characteristics of patients with peripheral venous catheter-associated and central venous catheter-associated bloodstream infections

\begin{tabular}{|c|c|c|c|}
\hline & PVC-BSI $(n=124)$ & CVC-BSI $(n=110)$ & $p$ \\
\hline Age (mean, [range]) & $66[18-95]$ & $65[19-91]$ & 0.532 \\
\hline $\operatorname{Sex}(M: F)$ & $88: 36$ & $77: 33$ & 0.887 \\
\hline Length of hospitalization (days; median [range]) & $16[2-9399]$ & $28[3-339]$ & $<0.001$ \\
\hline Ward type & & & 0.114 \\
\hline Medicine & 58 & 58 & \\
\hline Surgery & 44 & 40 & \\
\hline Emergency Department & 6 & 0 & \\
\hline ICU & 16 & 12 & \\
\hline Length of catheter indwelling (days; median, [range]) & $3[1-13]$ & $16[2-133]$ & $<0.001$ \\
\hline Mechanical ventilation (\# patients) & $11(8.9 \%)$ & $8(7.3 \%)$ & 0.347 \\
\hline Age-adjusted Charlson score (mean, [range]) & $6[0-15]$ & $6[1-13]$ & 0.435 \\
\hline Myocardial infarction & 8 & 7 & \\
\hline Congestive heart failure & 10 & 12 & \\
\hline Peripheral vascular disorder & 2 & 2 & \\
\hline Cerebrovascular disease & 15 & 7 & \\
\hline Dementia & 15 & 1 & \\
\hline Chronic obstructive lung disease & 5 & 5 & \\
\hline Connective tissue disease & 9 & 8 & \\
\hline Peptic ulcer disease & 3 & 3 & \\
\hline Diabetes mellitus & 28 & 29 & \\
\hline Chronic kidney disease & 5 & 2 & \\
\hline Hemiplegia & 13 & 3 & \\
\hline Leukemia & 11 & 15 & \\
\hline Malignant lymphoma & 2 & 17 & \\
\hline Solid tumor & 50 & 46 & \\
\hline Liver disease & 24 & 9 & \\
\hline AIDS & 1 & 0 & \\
\hline Pre-BSI SOFA score (median [range]) & $1[0-11]$ & $1[0-15]$ & 0.758 \\
\hline Change in SOFA score after BSI (median, [range]) & $+1[-1$ to +10$]$ & $+1[-2$ to +11$]$ & 0.690 \\
\hline
\end{tabular}

$P V C$-BSI peripheral venous catheter-blood stream infection, $C V C$-BSI central venous catheter-blood stream infection, $M$ male, $F$ female, $B S I$ blood stream infection, ICU Intensive Care Unit, SOFA sequential organ failure assessment 
Table 2 Distribution of pathogens in patients with peripheral venous catheter-associated and central venous catheter-associated bloodstream infections

\begin{tabular}{llll}
\hline & $\begin{array}{l}\text { PVC-BSI } \\
(\boldsymbol{n}=\mathbf{1 2 4})\end{array}$ & $\begin{array}{l}\text { CVC-BSI } \\
(\boldsymbol{n}=\mathbf{1 1 0})\end{array}$ & $\boldsymbol{p}$ \\
\hline $\begin{array}{l}\text { S. aureus Oxacillin } \\
\text { resistance rate }\end{array}$ & $44(23 \%)$ & $21(33 \%)$ & 0.006 \\
$\begin{array}{l}\text { CNS }{ }^{\mathrm{a}} \text { Oxacillin- } \\
\text { resistance rate }\end{array}$ & $35(80 \%)$ & $49(84 \%)$ & 0.010 \\
$\begin{array}{l}\text { Enterococcus } \\
\text { Gram-negative bacilli } \\
\quad \text { glucose non- }\end{array}$ & $24(8)$ & $9(3)$ & 0.015 \\
$\quad \begin{array}{l}\text { fermentative } \\
\text { bacteria) }\end{array}$ & & 3 & \\
$\begin{array}{l}\text { Candida } \\
\text { Polymicrobial }\end{array}$ & 2 & 21 & 0.668 \\
Others & 11 & 4 & 0.507 \\
\hline
\end{tabular}

PVC-BSI Peripheral venous catheter-associated blood stream infection, $C V C$-BSI central venous catheter-associated blood stream infection

${ }^{a}$ CNS coagulase-negative staphylococci

b Bacillus sp. (4 cases), Corynebacterium sp. (1 case), and Mycobacterium sp. (1 case)

c Streptococcus agalactiae (1 case), Corynebacterium sp. (1 case), and Micrococcus sp. (1 case)

\section{DISCUSSION}

In general, the incidence rates of PVC-BSIs were far less than CVC-BSIs [5-7]. However, PVCs were much more frequently used than CVCs $[8,9]$, and, therefore, the absolute number of PVC-BSIs surpassed that of CVC-BSIs. This study showed that the overall clinical outcomes did not differ between patients with CVC-BSIs and PVC-BSIs. However, the distribution of the causative pathogens differed between the two groups, indicating that PVCs are not safer than CVCs with regards to BSIs.

The length of catheter indwelling required to develop a PVC-BSI was far shorter than that required to develop a CVC-BSI. The Infectious Diseases Society of America guidelines for the prevention of intravascular catheter-related infections [20] state "there is no need to replace peripheral catheters more frequently than every $72-96 \mathrm{~h}$ to reduce the risk of infection and phlebitis in adults". Based on this guideline, PVCs were replaced every $72-96 \mathrm{~h}$ in our hospital, and, therefore, the median length of PVC indwelling was 3 days. As many as 68 cases (55\%) of PVC-BSI developed within 3 days of indwelling, increasing to 123 cases (99\%) within 10 days of indwelling. In contrast, only 35 cases $(32 \%)$ of CVC-BSI developed within 10 days of indwelling. Colonization and biofilm formation occurs within 3 days of indwelling [21]. Molecular methods have shown frequent extraluminal and intraluminal bacterial colonization in PVCs, even though culture methods detected no bacteria [22]. Biofilms tend to form on the external surface and not in the internal lumen of the catheter for up to 10 days $[23,24]$. Based on these results and reports, it is believed that the main mechanism of PVC-BSI development involves contamination outside the catheter, mainly from the skin during the insertion procedure. The Catalonian Nosocomial Infection Surveillance (VINCat) program reported the incidence of PVC-BSIs and CVCBSIs to be 0.05 and $0.10-0.14$ per 1000 patientdays, respectively, in the non-ICU wards. The incidence of PVC-BSIs in our study was slightly higher than that in the VINCat program. Therefore, cleaning of the skin using aseptic techniques before insertion of PVC is critical for reducing the incidence of PVC-BSIs; however, replacing the catheters more frequently than every $72-96 \mathrm{~h}$ does not help.

Our findings demonstrated a difference in the distribution of pathogen species between the two groups. In line with previous reports [25-27], we found that 49 (45\%) CVC-BSI cases were caused by coagulase-negative staphylococci. Contrastingly, $S$. aureus was the most common causative pathogen in PVC-BSI cases, and Gram-negative bacilli were not observed less frequently than coagulasenegative staphylococci. Our findings on causative pathogens in PVC-BSI cases were consistent with those reported previously $[6,10,28]$.The duration of $S$. aureus-associated bacteremia is reported to be significantly longer in the PVC-BSI group than in the non-PVC-BSI group [29], highlighting the fact that PVC-BSIs are a major source of $S$. aureus- 
Table 3 Treatment of peripheral venous catheter-associated and central venous catheter-associated bloodstream infections

\begin{tabular}{llll}
\hline & PVC-BSI $(\boldsymbol{n}=\mathbf{1 2 4})$ & CVC-BSI $(\boldsymbol{n}=\mathbf{1 1 0})$ & $\boldsymbol{p}$ \\
\hline Catheter removal within 24 h & 114 & 87 & 0.008 \\
Days to catheter removal after blood culture & $0[0-4]$ & $0[0-18]$ & 0.006 \\
Appropriate treatment on the first day & $49 \%$ & $37 \%$ & 0.086 \\
Treatment regimen on the first day & & & 0.068 \\
$\beta$-lactams & 70 & 67 & \\
$\beta$-lactams + glycopeptides & 6 & 13 & \\
Glycopeptides & 5 & 4 & 0.104 \\
Others & 10 & 11 & 0.001 \\
No antibiotics & 33 & 15 & \\
Delay in appropriate treatment (days; median, [range]) & $1[0-11]$ & $10-6]$ & \\
Definitive treatment regimen & & 24 & \\
$\beta$-lactams & 57 & 33 & \\
$\beta$-lactams + glycopeptides & 19 & 25 & \\
Glycopeptides & 34 & 25 & 3 \\
Others & 10 & $14[0-71]$ & \\
No treatment & 4 & $13[0-65]$ & \\
Duration of antimicrobial therapy (days; median, [range]) & & \\
\hline
\end{tabular}

$P V C-B S I$ peripheral venous catheter-associated blood stream infection, $C V C-B S I$ central venous catheter-associated blood stream infection

associated bacteremia in clinical settings. To treat $S$. aureus infections, glycopeptides are important agents used for empirical therapy until the blood culture results are available [30]. $\beta$-lactams may also be important for the treatment of PVC-BSIs because of the dominance of Gram-negative bacilli. It has recently been reported that Gramnegative rods were more frequently identified in PVC-BSIs than in CVC-BSIs [31]. However, it is necessary to be careful while interpreting these results due to differences in the antibiograms for each setting, which decide the appropriate initial empirical therapy.

The third point is the prognosis of PVC-BSIs. Consistent with a previous study that showed comparable overall mortality between the CVC and PVC groups [8, 32], we also found that the 30-day all-cause mortality was comparable between the two groups. While some studies have reported a deterioration in prognoses of critically ill patients in ICUs due to CVC-BSIs [1, 3, 33], another study concluded that CVC-BSIs had no influence on the prognosis [34]. These studies may indicate that the impact of CRBSIs on mortality depends on the severity of the patient's condition. Thus, the prognoses and the distribution of pathogen species between CVC-BSIs and PVCBSIs were consistent with other reports.

Our study has some limitations. First, there is a possibility of over or under diagnosis of CRBSIs, since catheter-tip culture information was not used. Second, as the lengths of hospitalization and catheter indwelling were shorter in the PVC-BSI group, there could be a slight bias in patient selection due to the retrospective nature of the study. Third, pathogen species distribution may vary depending on the facility. Finally, this study focused on cases from 2011 to 


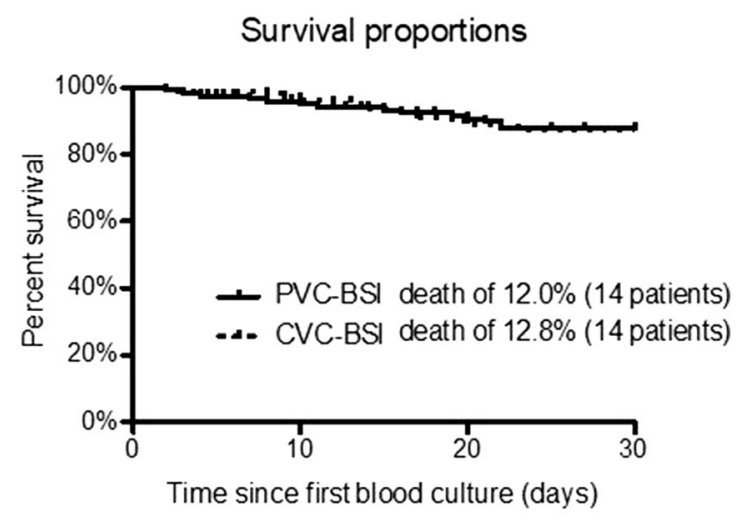

Number of subjects

$\begin{array}{lllll}\text { PVC-BSI } & 124 & 120 & 111 & 103\end{array}$

$\begin{array}{llll}\text { CVC-BSI } & 110 & 109 & 108\end{array}$

Fig. 2 Survival in patients with catheter-related bloodstream infections, showing the curves for the 30-day allcause mortality among patients with peripheral venous catheter-associated and central venous catheter-associated bloodstream infections. The all-cause mortality at 30 days was $12.0 \%$ (14 patients) for the PVC-BSI group and $12.8 \%$ (14 patients) for the CVC-BSI group PVC-BSI peripheral venous catheter-associated blood stream infection, $C V C$ BSI central venous catheter-associated blood stream infection

2013; the findings might be different today. Further studies will be needed to determine the clinical impact of PVC-BSIs on patient safety so that PVC-BSIs can be managed optimally.

\section{CONCLUSIONS}

Our findings suggest that while pathogen species distribution varies between PVC-BSIs and CVC-BSIs, the all-cause mortality rate did not differ significantly between the two groups. Because PVCs are more frequently used in hospital settings, their absolute contribution to nosocomial infections could be comparable to that of CVCs. Therefore, PVC-BSIs should be given the same attention that is given to CVCBSIs in order to prevent their incidence.

\section{ACKNOWLEDGEMENTS}

We would like to thank all the participants in this study.
Funding. No funding or sponsorship was received for this study or publication of this article. The Rapid Service Fee was funded by the authors.

Authorship. All named authors meet the International Committee of Medical Journal Editors (ICMJE) criteria for authorship for this article, take responsibility for the integrity of the work as a whole, and have given their approval for this version to be published.

Prior Presentation. This work was presented at the Interscience Conference on Antimicrobial Agents and Chemotherapy (ICAAC) 2015, September 17-21, 2015, San Diego, USA.

Disclosures. Keita Tatsuno, Mahoko Ikeda, Yoshitaka Wakabayashi, Shintaro Yanagimoto, Shu Okugawa and Kyoji Moriya have nothing to declare.

Compliance with Ethics Guidelines. The study was approved by the ethics committee of Graduate School of Medicine and Faculty of Medicine, The University of Tokyo (\#3538). The requirement for written informed consent was waived due to the observational retrospective nature of the study.

Data Availability. The datasets during and analyzed during the current study are available from the corresponding author on reasonable request.

Open Access. This article is distributed under the terms of the Creative Commons Attribution-NonCommercial 4.0 International License (http://creativecommons.org/licenses/ by-nc/4.0/), which permits any noncommercial use, distribution, and reproduction in any medium, provided you give appropriate credit to the original author(s) and the source, provide a link to the Creative Commons license, and indicate if changes were made.

\section{REFERENCES}

1. Renaud B, Brun-Buisson C, Group IC-BS. Outcomes of primary and catheter-related bacteremia. A 
cohort and case-control study in critically ill patients. Am J Respir Crit Care Med. 2001;163(7):1584-90.

2. Brunelli SM, Turenne W, Sibbel S, Hunt A, Pfaffle A. Clinical and economic burden of bloodstream infections in critical care patients with central venous catheters. J Crit Care. 2016;35:69-74.

3. Stevens V, Geiger K, Concannon C, Nelson RE, Brown J, Dumyati G. Inpatient costs, mortality and 30-day re-admission in patients with central-lineassociated bloodstream infections. Clin Microbiol Infect. 2014;20(5):O318-24.

4. Guembe M, Perez-Granda MJ, Capdevila JA, Barberan J, Pinilla B, Martin-Rabadan P, et al. Nationwide study on the use of intravascular catheters in internal medicine departments. J Hosp Infect. 2015;90(2):135-41.

5. Fakih MG, Jones K, Rey JE, Berriel-Cass D, Kalinicheva T, Szpunar S, et al. Sustained improvements in peripheral venous catheter care in non-intensive care units: a quasi-experimental controlled study of education and feedback. Infect Control Hosp Epidemiol. 2012;33(5):449-55.

6. Coello R, Charlett A, Ward V, Wilson J, Pearson A, Sedgwick J, et al. Device-related sources of bacteraemia in English hospitals-opportunities for the prevention of hospital-acquired bacteraemia. J Hosp Infect. 2003;53(1):46-57.

7. Maki DG, Kluger DM, Crnich CJ. The risk of bloodstream infection in adults with different intravascular devices: a systematic review of 200 published prospective studies. Mayo Clin Proc. 2006;81(9):1159-71.

8. Pujol M, Hornero A, Saballs M, Argerich MJ, Verdaguer R, Cisnal M, et al. Clinical epidemiology and outcomes of peripheral venous catheter-related bloodstream infections at a university-affiliated hospital. J Hosp Infect. 2007;67(1):22-9.

9. Zingg W, Pittet D. Peripheral venous catheters: an under-evaluated problem. Int J Antimicrob Agents. 2009;34(Suppl 4):S38-42.

10. Freixas N, Bella F, Limon E, Pujol M, Almirante B, Gudiol F. Impact of a multimodal intervention to reduce bloodstream infections related to vascular catheters in non-ICU wards: a multicentre study. Clin Microbiol Infect. 2013;19(9):838-44.

11. Lee WL, Chen HL, Tsai TY, Lai IC, Chang WC, Huang $\mathrm{CH}$, et al. Risk factors for peripheral intravenous catheter infection in hospitalized patients: a prospective study of 3165 patients. Am J Infect Control. 2009;37(8):683-6.
12. Bregenzer $\mathrm{T}$, Conen $\mathrm{D}$, Sakmann $\mathrm{P}$, Widmer AF. Is routine replacement of peripheral intravenous catheters necessary? Arch Intern Med. 1998;158(2):151-6.

13. Curran ET, Coia JE, Gilmour H, McNamee S, Hood J. Multi-centre research surveillance project to reduce infections/phlebitis associated with peripheral vascular catheters. J Hosp Infect. 2000;46(3):194-202.

14. Tagalakis V, Kahn SR, Libman M, Blostein M. The epidemiology of peripheral vein infusion thrombophlebitis: a critical review. Am J Med. 2002;113(2):146-51.

15. Malach T, Jerassy Z, Rudensky B, Schlesinger Y, Broide E, Olsha O, et al. Prospective surveillance of phlebitis associated with peripheral intravenous catheters. Am J Infect Control. 2006;34(5):308-12.

16. Horan TC, Andrus M, Dudeck MA. CDC/NHSN surveillance definition of health care-associated infection and criteria for specific types of infections in the acute care setting. Am J Infect Control. 2008;36(5):309-32.

17. Vincent JL, Moreno R, Takala J, Willatts S, De Mendonca A, Bruining $\mathrm{H}$, et al. The SOFA (Sepsisrelated Organ Failure Assessment) score to describe organ dysfunction/failure. On behalf of the Working Group on Sepsis-Related Problems of the European Society of Intensive Care Medicine. Intensive Care Med. 1996;22(7):707-10.

18. Charlson ME, Pompei P, Ales KL, MacKenzie CR. A new method of classifying prognostic comorbidity in longitudinal studies: development and validation. J Chronic Dis. 1987;40(5):373-83.

19. Charlson M, Szatrowski TP, Peterson J, Gold J. Validation of a combined comorbidity index. J Clin Epidemiol. 1994;47(11):1245-51.

20. O'Grady NP, Alexander M, Burns LA, Dellinger EP, Garland J, Heard SO, et al. Guidelines for the prevention of intravascular catheter-related infections. Clin Infect Dis. 2011;52(9):e162-93.

21. Anaissie E, Samonis G, Kontoyiannis D, Costerton J, Sabharwal U, Bodey G, et al. Role of catheter colonization and infrequent hematogenous seeding in catheter-related infections. Eur J Clin Microbiol Infect Dis. 1995;14(2):134-7.

22. Zhang L, Morrison M, Nimmo GR, Sriprakash KS, Mondot S, Gowardman JR, et al. Molecular investigation of bacterial communities on the inner and outer surfaces of peripheral venous catheters. Eur J Clin Microbiol Infect Dis. 2013;32(8):1083-90. 
23. Raad I, Costerton W, Sabharwal U, Sacilowski M, Anaissie E, Bodey GP. Ultrastructural analysis of indwelling vascular catheters: a quantitative relationship between luminal colonization and duration of placement. J Infect Dis. 1993;168(2):400-7.

24. Donlan RM, Costerton JW. Biofilms: survival mechanisms of clinically relevant microorganisms. Clin Microbiol Rev. 2002;15(2):167-93.

25. Bouza E, San Juan R, Munoz P, Pascau J, Voss A, Desco $\mathrm{M}$, et al. A European perspective on intravascular catheter-related infections: report on the microbiology workload, aetiology and antimicrobial susceptibility (ESGNI-005 Study). Clin Microbiol Infect. 2004;10(9):838-42.

26. Marschall J, Leone C, Jones M, Nihill D, Fraser VJ, Warren DK. Catheter-associated bloodstream infections in general medical patients outside the intensive care unit: a surveillance study. Infect Control Hosp Epidemiol. 2007;28(8):905-9.

27. Kang J, Sickbert-Bennett EE, Brown VM, Weber DJ, Rutala WA. Changes in the incidence of health care-associated pathogens at a university hospital from 2005 to 2011. Am J Infect Control. 2014;42(7):770-5.

28. Sato A, Nakamura I, Fujita H, Tsukimori A, Kobayashi T, Fukushima S, et al. Peripheral venous catheter-related bloodstream infection is associated with severe complications and potential death: a retrospective observational study. BMC Infect Dis. 2017;17(1):434.
29. Austin ED, Sullivan SB, Whittier S, Lowy FD, Uhlemann AC. Peripheral intravenous catheter placement is an underrecognized source of Staphylococcus aureus bloodstream infection. Open Forum Infect Dis. 2016;3(2): ofw072.

30. Mermel LA, Allon M, Bouza E, Craven DE, Flynn P, $\mathrm{O}^{\prime}$ Grady NP, et al. Clinical practice guidelines for the diagnosis and management of intravascular catheter-related infection: 2009 Update by the Infectious Diseases Society of America. Clin Infect Dis. $2009 ; 49(1): 1-45$.

31. Tsuboi M, Hayakawa K, Mezaki K, Katanami Y, Yamamoto K, Kutsuna S, et al. Comparison of the epidemiology and microbiology of peripheral lineand central line-associated bloodstream infections. Am J Infect Control. 2019;47(2):208-10.

32. Saliba P, Hornero A, Cuervo G, Grau I, Jimenez E, Garcia D, et al. Mortality risk factors among nonICU patients with nosocomial vascular catheter-related bloodstream infections: a prospective cohort study. J Hosp Infect. 2018;99(1):48-54.

33. Warren DK, Quadir WW, Hollenbeak CS, Elward AM, Cox MJ, Fraser VJ. Attributable cost of catheterassociated bloodstream infections among intensive care patients in a nonteaching hospital. Crit Care Med. 2006;34(8):2084-9.

34. Blot SI, Depuydt P, Annemans L, Benoit D, Hoste E, De Waele JJ, et al. Clinical and economic outcomes in critically ill patients with nosocomial catheterrelated bloodstream infections. Clin Infect Dis. 2005;41(11):1591-8. 